\title{
Importancia de los hábitos de estudio en el rendimiento académico del adolescente: diferencias por género
}

\section{Importance of study habits on adolescents' academic achievement: gender differences}

\author{
Ana Capdevila Seder ${ }^{1}$ \\ acapdevi@uji.es \\ Héctor Bellmunt Villalonga \\ bellmunt@uji.es \\ Universitat Jaume I de Castellón, España
}

\section{Resumen:}

Los hábitos de estudio juegan un papel fundamental en el desempeño académico, pero éstos no se miden únicamente por la cantidad de tiempo dedicado a estudiar, es más bien una cuestión de calidad del estudio. En el presente artículo se analizan en profundidad los hábitos de estudio de 203 adolescentes de $2^{\circ}$ ciclo de ESO de centros públicos de Castellón de la Plana. Los instrumentos utilizados son el cuestionario CHTE (Cuestionario de Hábitos y Técnicas de Estudio) y las calificaciones escolares. El objetivo es detectar qué aspectos de los hábitos de estudio se relacionan con el rendimiento académico, así como hallar diferencias por género. Aspectos como la planificación del tiempo, la actitud o el lugar de estudio, se presentan como elementos importantes a tener en cuenta de cara a mejorar el rendimiento académico. Existen importantes diferencias por género, tanto en hábitos de estudio como en el rendimiento académico.

\begin{abstract}
:
Study habits play a major role in academic achievement, but these are not measured only by the amount of time spent studying, it is rather a matter of study quality. In this article the study habits of 203 adolescents from 2nd ESO from Castellón de la Plana state schools are analyzed in depth. The instruments used are the CHTE (habits and study skills) and school grades. The aim is to identify which aspects of study habits are related to academic achievement, and to find gender differences. Aspects such as time planning, attitude or place of study are presented as important elements to consider in order to improve academic achievement. There are significant gender differences in both study habits and academic achievement.
\end{abstract}

1 Dirección para correspondencia (correspondence address):

Ana Capdevila Seder. Dpto. de Educación. Facultad de Ciencias Humanas y Sociales. Universitat Jaume I de Castelló. Campus de Riu Sec. 12071 Castellón (España). 
Importancia de los hábitos de estudio en el rendimiento académico del adolescente: diferencias por género

Ana Capdevila Seder y Héctor Bellmunt Villalonga

Palabras clave:

Rendimiento académico; hábitos de estudio; género.

\section{Key words:}

Academic achievement; study habits; gender.

\section{Résumé:}

Les habitudes d'étude jouent un rôle important dans le rendement scolaire, mais celles-ci ne sont pas mesurées seulement par la quantité de temps passé à étudier, il s'agit plutôt de la qualité de l'étude. Dans cet article, nous analysons en profondeur les habitudes d'étude de 203 adolescents de 2e cycle de l'ESO dans des établissements publiques à Castellón de la Plana. Les instruments utilisés sont les CHTE (Enquête d'Habitudes et Techniques d'Etude) et les notes aux évaluations scolaires. L'objectif est d'identifier les aspects des habitudes d'étude qui sont liés au rendement scolaire, et de percevoir de possibles différences entre sexes. Des aspects tels que la planification du temps, l'attitude ou le lieu d'étude sont présentés comme des éléments importants à prendre en compte pour améliorer le rendement scolaire. II existe des différences significatives entre les sexes dans les habitudes d'étude et le rendement scolaire.

\section{Mots clés:}

Rendement scolaire; habitudes d'étude; sexe.

Fecha de recepción: 2-5-2014

Fecha de aceptación: 4-12-2015

\section{Introducción}

Actualmente resulta de gran interés el estudio de aquellos aspectos que puedan influir de una manera u otra en el rendimiento académico del alumnado adolescente. El conocimiento de los factores que pueden incidir en un mejor rendimiento de nuestros alumnos es una tarea primordial si se quiere diseñar programas de intervención que vayan en la dirección de prevenir el fracaso escolar (Barbero, Holgado, \& Chacón, 2007).

Según datos de la Subdirección General de Estadística y Estudios del Ministerio (SGEEM, 2012), España está en la cola en varios de los indicadores internacionales de la educación. En cuanto al porcentaje de abandono temprano de la Educación Secundaria, la media europea es del 14,1\%, siendo en España del 28,4\%, superado sólo por Portugal $(28,7 \%)$ y Malta $(39,6 \%)$. Además del abandono escolar, el bajo rendimiento académico también es tema de preocupación. Teniendo en cuenta las principales conclusiones del último informe PISA (PISA, 2012), la Organización para la Cooperación y el Desarrollo Económicos (OCDE) indica que el rendimiento medio en España está por debajo de la media y no ha mejorado desde hace 10 años. Así, muchos autores y autoras han centrado sus investigaciones en aspectos relacionados con el éxito y el 
fracaso escolar (Fullana, 2008; González, 2003; Molina y García, 1984; Perrenoud, 1990; Wall, 1970).

Tras una revisión de diferentes definiciones del rendimiento académico, Fullana (2008) concluye que es un resultado del proceso de aprendizaje escolar, resultado en el cual convergen los efectos de numerosas variables sociales, personales, y sus interrelaciones. En general, se utilizan los expedientes académicos y las calificaciones de los escolares como fuente principal para valorar los resultados de la enseñanza; hoy por hoy, constituyen el criterio que existe para definir el rendimiento académico (Corea, 2001). Asimismo, autoras y autores como Córdoba (2010) o Moriana et al. (2006), utilizaron la nota media de las calificaciones finales como forma de medir el rendimiento académico en alumnado de Educación Secundaria.

Las variables que inciden sobre el rendimiento académico son tantas que, normalmente, en las investigaciones se opta por simplificar la búsqueda, enfocándolas en el estudio de las relaciones de una o dos variables con el rendimiento escolar (Fullana, 2008). Aunque los resultados difieren de unas investigaciones a otras, por lo general hay un grupo de variables que aparecen con mayor frecuencia en la mayoría de estudios, como son por ejemplo: género, edad, entorno sociocultural, tipo de centro educativo, hábitos de estudio, hábitos de lectura y hábitos de ocio. En todos estos casos se considera al rendimiento académico como variable dependiente de estas otras variables, con lo que se establece una relación de causa/efecto. En este trabajo se ha acotado el número de variables, teniendo en cuenta el género y los hábitos de estudio como variables determinantes del rendimiento académico.

La Real Academia Española de la Lengua (2007) define hábito como "modo especial de proceder o conducirse adquirido por repetición de actos iguales o semejantes u originados por tendencias instintivas". Hernández y García (1991) dicen que los hábitos son los comportamientos que se tienen automatizados, que se realizan sin pensar en ellos, porque se han ido adquiriendo a lo largo del tiempo tras numerosas repeticiones. Los hábitos facilitan y favorecen la ejecución de cualquier actividad porque permiten que se realice con mayor rapidez, eficacia y precisión, a la vez que disminuyen el esfuerzo físico y psicológico necesario para su correcta realización. Con esto, se aprecia la importancia que tiene el convertir una acción deseada en hábito, para facilitar así su realización; y la forma de hacer que esto suceda, se consigue mediante la repetición de dicha acción deseada. 
Importancia de los hábitos de estudio en el rendimiento académico del adolescente: diferencias por género

Ana Capdevila Seder y Héctor Bellmunt Villalonga

Cuando se trata el tema de los hábitos de estudio puede generarse cierta confusión al utilizar diferentes términos al respecto. Es común el uso tanto de hábitos de estudio, como de técnicas de estudio, métodos de estudio, estrategias de aprendizaje, o simplemente se utiliza el término estudiar. En referencia a esto, Hernández y García (1991) definen método de estudio como el conjunto más o menos sistemático de pautas, que dan luz a la tarea de estudio y que se especifica en estrategias concretas, como suelen ser el uso de subrayados, cuadros sinópticos y reglas nemotécnicas. Por otra parte, Báez y Báez (1998), afirman que estudiar es el modo particular que se tiene de aprender, el proceso que se sigue para adquirir y demostrar, casi siempre mediante exámenes, que se tienen los conocimientos necesarios respecto a unas asignaturas. Con lo que estudiar, además de memorizar, es ir a clase, buscar bibliografía, hacer trabajos, acudir a bibliotecas, pedir ayuda al profesorado, padres, madres, compañeras, compañeros, etc.

Dada toda esta diversidad de conceptos y para evitar confusiones en la terminología, en este texto se hablará de hábitos de estudio como algo general, que engloba tanto a las técnicas como a las estrategias o los métodos de estudio.

En cuanto a la organización o planificación del tiempo, dentro de los hábitos de estudio, ésta debe ser flexible y se ha de estructurar en torno a diversas actividades (Corea, 2001). Tan importante es el aspecto organizativo dentro de los hábitos de estudio, que García (1988) los define como "el resultado de una ordenación previa de la actividad o planificación y cumplimiento continuo de un plan propuesto".

Además de la organización del tiempo, los factores psicológicos como la actitud o la motivación, también se destacan como importantes a tener en cuenta en la preparación antes del estudio (Báez y Báez, 1998). En base a esto, Amrai, Motlagh, Zalani y Parhon (2011), hallaron una correlación positiva entre la motivación académica y el rendimiento académico. Estos autores creen que muchos estudiantes, hombres y mujeres, no saben estudiar, pero, quizás muchos y muchas más son quienes encuentran dificultades en sentir motivación hacia el estudio.

Vista la importancia de los hábitos de estudio, numerosos estudios los relacionan directamente con los resultados académicos de los sujetos en edad escolar; encontrando una relación positiva entre ambos (Díaz, 1995; Fullana, 2008; Hernández y García, 1991; Martínez-Otero, 1996; Meneghetti, De Beni y Cornoldi, 2007; Nonis y Hudson, 2010). 
Por otra parte, si la relación que se busca es entre el tiempo dedicado al estudio y el rendimiento académico, los resultados varían de unas investigaciones a otras, de forma que Corea (2001) y Plant, Ericsson, Hill y Asberg (2005) no encuentran conexión alguna entre ambas variables. Sin embargo, Córdoba (2010) halla una relación positiva. Por último, investigaciones realizadas por Amrai et al. (2011) y Salas (1999) se centran en la motivación académica, afirmando que ésta se relaciona de forma positiva con el rendimiento académico.

En cuanto al género como variable, por lo general, las investigaciones coinciden en afirmar que hay una tendencia por parte de las mujeres a obtener mejores calificaciones escolares que los hombres (Córdoba, 2010; Corea, 2001; Kovacs et al., 2008; Ruiz de Miguel, 2009; Yu, Chan, Cheng, Sung, y Hau, 2006), siendo incluso una variable predictora del rendimiento (Crosnoe, 2002). Por otro lado, los resultados de Grimes (1985), quien analizó las diferencias de género en la utilización de estrategias de aprendizaje, indican que las alumnas obtienen mayor puntuación que los alumnos en las escalas de actitud, motivación, administración de tiempo, ayudas de estudio y autocomprobación. Por su lado, Núñez, González, García, González y García (1995), con una muestra de estudiantes de 10 a 14 años, encontraron diferencias significativas a favor de las alumnas en las escalas de motivación, ayudas de estudio y ansiedad.

Así, en el presente estudio se plantearon dos objetivos:

1. Analizar los hábitos de estudio y su relación con el rendimiento académico de adolescentes de $2^{\circ}$ ciclo de ESO.

2. Analizar los hábitos de estudio y el rendimiento académico en busca de diferencias por género, en adolescentes de $2^{\circ}$ ciclo de ESO.

\section{Método}

\section{Participantes}

Este estudio ha sido realizado sobre una muestra total de 203 sujetos cuyas edades oscilan entre 14 y 18 años $(M=15,04 D T=.86) ; 114$ hombres y 89 mujeres. Además, 169 son de nacionalidad española y 34 de nacionalidad extranjera. Todos ellos estudiantes de un total de 9 centros 
Importancia de los hábitos de estudio en el rendimiento académico del adolescente: diferencias por género

Ana Capdevila Seder y Héctor Bellmunt Villalonga

públicos de Castellón de la Plana en los cursos escolares de $3^{\circ}$ y $4^{\circ}$ de ESO; las diferencias de edades se deben a la presencia de 15 alumnos y alumnas que habían repetido curso. Se seleccionó de forma aleatoria a los participantes de entre aquellos que de forma voluntaria quisieron participar. Se les explicó en qué consistía la investigación y se entregó una autorización paterna a los sujetos seleccionados.

\section{Instrumentos}

Cuestionario de Hábitos y Técnicas de Estudio (CHTE)

(Álvarez y Fernández, 2002)

El CHTE mide los hábitos y técnicas de estudio, con 56 ítems con categorías de respuestas dicotómicas (Sí/No). Se eligió este cuestionario por ser de rápida administración y por ajustarse a las necesidades de la investigación. Además de medir un factor general de hábitos de estudio (HE), también mide los hábitos en 7 escalas AC: Actitud general hacia el estudio, LU: Lugar de estudio, ES: Estado físico del escolar, PL: Planificación del tiempo, TE: Técnicas de estudio, EX: Exámenes y ejercicios y TR: Trabajos. Tras su corrección se obtiene una puntuación global de 0 a 100 y una puntuación específica para cada escala, también de 0 a 100.

Las notas académicas de los sujetos fueron proporcionadas por los propios centros educativos mediante las Actas Oficiales o el Boletín personal, según el caso. Corresponden a las notas finales de la $2^{\text {a }}$ evaluación del curso académico 2011-2012, que coincide con el periodo de recogida de datos. Para calcular la media de las notas, se han incluido todas las materias obligatorias del curso, es decir, se ha excluido la materia optativa en el caso de $3^{\circ}$ de ESO y las tres materias específicas de $4^{\circ}$ de ESO. De aquí se obtiene la variable RA (rendimiento académico).

\section{Procedimiento}

Para seleccionar la muestra, lo primero que se hizo fue conseguir los diferentes permisos para poder llevar a cabo la investigación. Los sujetos son menores de edad, por lo que se necesitaba autorización por varias vías: en primer lugar, la autorización de la Conselleria de Educación de la Generalitat Valenciana, seguida por un Informe favorable de la Comisión Deontológica de la Universitat Jaume I de Castelló. Después de obtuvo la autorización de cada centro educativo, firmada por la Dirección 
del mismo. Por último, el consentimiento informado por parte del padre o madre, tutor o tutora de cada sujeto.

La administración de los cuestionarios se llevó a cabo en el propio centro educativo, en horario escolar. Esta labor se hizo estando presente el investigador principal, explicando cómo cumplimentar el cuestionario y resolviendo las dudas que pudiesen surgir. El tiempo requerido para rellenar el cuestionario CHTE fue de aproximadamente 15 minutos.

\section{Análisis de datos}

Se realizó dos tipos de cálculos, dependiendo de la naturaleza de las variables objeto de estudio. Este análisis se basó en dos tipos de estadísticos: la correlación de Pearson y T de Student. En caso de que no se haya podido admitir una distribución normal (tras aplicar las pruebas de normalidad Kolmogorov-Smirnov y Shapiro-Wilk), se ha aplicado la oportuna prueba no paramétrica, en este caso la "U de Mann-Whitney". Se estableció un intervalo de confianza del 95\%.

Para el procesamiento de los datos y su tratamiento estadístico se utilizó el paquete estadístico PASW® Statistics versión 18 con licencia por la Universitat Jaume I de Castellón.

\section{Resultados}

\section{Descriptivos de variables continuas y correlaciones bivariadas}

En la Tabla 1, se presentan los estadísticos descriptivos de cada variable continua (media y desviación típica) y las correlaciones bivariadas entre cada una de ellas. 
Importancia de los hábitos de estudio en el rendimiento académico del adolescente: diferencias por género

ana Capdevila Seder y Héctor Bellmunt Villalonga

Tabla 1. Estadísticos descriptivos y correlaciones entre variables.

\begin{tabular}{|c|c|c|c|c|c|c|c|c|c|c|c|}
\hline Variables & $M$ & $D T$ & 1 & 2 & 3 & 4 & 5 & 6 & 7 & 8 & 9 \\
\hline 1. $\mathrm{RA}$ & 5.58 & 1.65 & - & $.33 * *$ & $.36^{* *}$ & .05 & $.46^{* *}$ & $.20^{* *}$ & .12 & $.18^{* *}$ & $.17^{*}$ \\
\hline 2. HE & 66.29 & 13.52 & - & - & $.73^{* *}$ & $.52 * *$ & $.77^{*}$ & $.54 * *$ & $.65^{* *}$ & $.65^{* *}$ & $.74 *$ \\
\hline 3. $\mathrm{PL}$ & 46.45 & 22.78 & - & - & - & $.28^{* *}$ & $.59^{* *}$ & $.31^{* *}$ & $.36^{* *}$ & $.35^{* *}$ & $.42^{* *}$ \\
\hline 4. ES & 67.90 & 20.17 & - & - & - & - & $.24^{* *}$ & $.21^{* *}$ & $.25^{* *}$ & $.19^{* *}$ & $.25^{* *}$ \\
\hline 5. AC & 71.53 & 18.96 & - & - & - & - & - & $.41^{* *}$ & $.40^{* *}$ & $.46^{* *}$ & $.48^{* *}$ \\
\hline 6. LU & 85.12 & 13.33 & - & - & - & - & - & - & $.31^{* *}$ & $.22 * *$ & $.29^{* *}$ \\
\hline 7. EX & 69.26 & 23.06 & - & - & - & - & - & - & - & $.24^{* *}$ & $.39 * *$ \\
\hline 8. TE & 64.92 & 21.42 & - & - & - & - & - & - & - & - & $.51^{* *}$ \\
\hline 9. TR & 58.87 & 22.69 & - & - & - & - & - & - & - & - & - \\
\hline
\end{tabular}

En cuanto al análisis correlacional, se observa que, teniendo en cuenta a toda la muestra, el rendimiento académico correlaciona de forma positiva y significativa con la puntuación global del cuestionario (HE), así como con las escalas del cuestionario PL, AC, LU, TE y TR; siendo la relación más fuerte la dada entre RA y AC (véase Tabla 1).

La puntuación en HE correlaciona de forma positiva con cada una de las siete escalas que lo componen.

Tabla 2. Estadísticos descriptivos y correlaciones entre variables en hombres.

\begin{tabular}{lccccccccccc}
\hline Variables & $M$ & $D T$ & 1 & 2 & 3 & 4 & 5 & 6 & 7 & 8 & 9 \\
\hline 1. RA & 5.31 & 1.64 & - & $.27^{* *}$ & $.30^{* *}$ & .07 & $.40^{* *}$ & $.21^{*}$ & .07 & .15 & .11 \\
2. HE & 63.86 & 14.20 & - & - & $.75^{* *}$ & $.52^{* *}$ & $.77^{* *}$ & $.56^{* *}$ & $.66^{* *}$ & $.62^{* *}$ & $.78^{* *}$ \\
3. PL & 45.61 & 23.76 & - & - & - & $.33^{* *}$ & $.60^{* *}$ & $.28^{* *}$ & $.35^{* *}$ & $.34^{* *}$ & $.50^{* *}$ \\
4. ES & 67.54 & 20.06 & - & - & - & - & $.27^{* *}$ & $.22^{*}$ & $.24^{*}$ & .13 & $.27^{* *}$ \\
5. AC & 67.46 & 20.34 & - & - & - & - & - & $.45^{* *}$ & $.35^{* *}$ & $.46^{* *}$ & $.49^{* *}$ \\
6. LU & 84.91 & 14.41 & - & - & - & - & - & - & $.35^{* *}$ & $.24^{*}$ & $.33^{* *}$ \\
7. EX & 65.09 & 23.92 & - & - & - & - & - & - & - & $.24^{*}$ & $.47^{* *}$ \\
8. TE & 61.89 & 21.82 & - & - & - & - & - & - & - & - & $.48^{* *}$ \\
9. TR & 54.54 & 23.50 & - & - & - & - & - & - & - & - & - \\
\hline
\end{tabular}

Nota. ${ }^{* *} p<.01 ; * p<.05 ; M=$ Media; $D T=$ Desviación típica; $\mathrm{RA}=$ rendimiento académico; $\mathrm{HE}=$ hábitos de estudio; $\mathrm{PL}=$ planificación; $\mathrm{ES}=$ estado físico; $\mathrm{AC}=$ actitud; $\mathrm{LU}=$ lugar de estudio; EX=preparación de exámenes; TE=técnicas de estudio; TR=realización de trabajos. 
Si tenemos en cuenta sólo a los hombres, el rendimiento académico correlaciona de forma positiva y significativa con la puntuación global del cuestionario $(\mathrm{HE})$, así como con las escalas del cuestionario $\mathrm{PL}, \mathrm{AC}$ y LU; siendo la relación más fuerte la dada entre RA y AC (véase Tabla 2). En este caso la puntuación en HE también correlaciona de forma positiva con cada una de las siete escalas que lo componen.

Tabla 3. Estadísticos descriptivos y correlaciones entre variables en mujeres.

\begin{tabular}{lccccccccccc}
\hline Variables & $M$ & $D T$ & 1 & 2 & 3 & 4 & 5 & 6 & 7 & 8 & 9 \\
\hline 1. RA & 5.92 & 1.61 & - & $.36^{* *}$ & $.46^{* *}$ & .03 & $.51^{* *}$ & .18 & .10 & .17 & .19 \\
2. HE & 69.40 & 11.99 & - & - & $.72^{* *}$ & $.55^{* *}$ & $.73^{*}$ & $.52^{* *}$ & $.60^{* *}$ & $.68^{* *}$ & $.64^{* *}$ \\
3. PL & 47.53 & 21.55 & - & - & - & $.22^{*}$ & $.60^{* *}$ & $.36^{* *}$ & $.36^{* *}$ & $.37^{* *}$ & $.29^{* *}$ \\
4. ES & 68.35 & 20.42 & - & - & - & - & .20 & $.21^{*}$ & $.26^{*}$ & $.27^{*}$ & $.24^{*}$ \\
5. AC & 76.74 & 15.65 & - & - & - & - & - & $.37^{* *}$ & $.42^{* *}$ & $.40^{* *}$ & $.36^{* *}$ \\
6. LU & 85.39 & 11.88 & - & - & - & - & - & - & $.25^{*}$ & .20 & $.24^{*}$ \\
7. EX & 74.61 & 20.84 & - & - & - & - & - & - & - & .18 & .18 \\
8. TE & 68.79 & 20.37 & - & - & - & - & - & - & - & - & $.51^{* *}$ \\
9. TR & 64.42 & 20.44 & - & - & - & - & - & - & - & - & - \\
\hline
\end{tabular}

Nota. ${ }^{* *} p<.01 ; * p<.05 ; M=$ Media; $D T=$ Desviación típica; $\mathrm{RA}=$ rendimiento académico; $\mathrm{HE}=$ hábitos de estudio; $\mathrm{PL}=$ planificación; $\mathrm{ES}=$ =stado físico; $\mathrm{AC}=$ actitud; $\mathrm{LU}=$ lugar de estudio; $E X=$ preparación de exámenes; $T E=$ técnicas de estudio; $\mathrm{TR}=$ realización de trabajos.

Si tenemos en cuenta sólo a las mujeres, el rendimiento académico correlaciona de forma positiva y significativa con la puntuación global del cuestionario (HE), así como con las escalas del cuestionario PL y AC; siendo la relación más fuerte la dada entre RA y AC (véase Tabla 3). En este caso la puntuación en HE también correlaciona de forma positiva con cada una de las siete escalas que lo componen. 
Importancia de los hábitos de estudio en el rendimiento académico del adolescente: diferencias por género

Ana Capdevila Seder y Héctor Bellmunt Villalonga

Tabla 4. Diferencias por género.

\begin{tabular}{lccccc}
\hline Prueba & \multicolumn{2}{c}{ Medias } & Estadístico & $\begin{array}{c}\text { Signifi- } \\
\text { cación }\end{array}$ & Resultado \\
\hline & Hombres & Mujeres & & & \\
\hline $\begin{array}{l}\text { Diferencias RA } \\
\text { género }\end{array}$ & 5.31 & 5.92 & T-Student & $.009^{* *}$ & Mujeres mejor RA \\
$\begin{array}{l}\text { Diferencias HE } \\
\text { género }\end{array}$ & 63.86 & 69.4 & T-Student & $.004^{* *}$ & Mujeres mejor HE \\
\hline $\begin{array}{l}\text { Diferencias AC } \\
\text { género }\end{array}$ & 67.46 & 76.74 & $\begin{array}{c}\text { U de Mann- } \\
\text { Whitney }\end{array}$ & $.001^{* *}$ & Mujeres mejor AC \\
\hline $\begin{array}{l}\text { Diferencias EX } \\
\text { género }\end{array}$ & 65.09 & 74.61 & $\begin{array}{c}\text { U de Mann- } \\
\text { Whitney }\end{array}$ & $.005^{* *}$ & Mujeres mejor EX \\
\hline $\begin{array}{l}\text { Diferencias TE } \\
\text { género }\end{array}$ & 61.89 & 68.79 & $\begin{array}{c}\text { U de Mann- } \\
\text { Whitney }\end{array}$ & $.027^{*}$ & Mujeres mejor TE \\
$\begin{array}{l}\text { Diferencias TR } \\
\text { género }\end{array}$ & 54.53 & 64.42 & $\begin{array}{c}\text { U de Mann- } \\
\text { Whitney }\end{array}$ & $.002^{* *}$ & Mujeres mejor TR \\
\hline $\begin{array}{l}\text { Diferencias PL } \\
\text { género }\end{array}$ & 45.61 & 47.53 & $\begin{array}{c}\text { U de Mann- } \\
\text { Whitney }\end{array}$ & .453 & Sin diferencias \\
\hline $\begin{array}{l}\text { Diferencias ES } \\
\text { género }\end{array}$ & 67.54 & 68.35 & $\begin{array}{c}\text { U de Mann- } \\
\text { Whitney }\end{array}$ & .843 & Sin diferencias \\
\hline $\begin{array}{l}\text { Diferencias LU } \\
\text { género }\end{array}$ & 84.91 & 85.39 & $\begin{array}{c}\text { U de Mann- } \\
\text { Whitney }\end{array}$ & .768 & Sin diferencias \\
\hline
\end{tabular}

Nota. ${ }^{* *} p<.01 ; * p<.05 ; \mathrm{RA}=$ rendimiento académico; HE=hábitos de estudio; $\mathrm{AC}=$ actitud; $E X=$ preparación de exámenes; $\mathrm{TE}=$ técnicas de estudio; $\mathrm{TR}=$ realización de trabajos; $\mathrm{PL}=$ planificación; $\mathrm{ES}=$ =stado físico; $\mathrm{LU}=$ lugar de estudio.

Al observar las diferencias en la media del rendimiento académico por género (véase Tabla 4) se ve que, de forma estadísticamente significativa, las mujeres tienen una media superior a los hombres en 0,6 puntos sobre 10. Las mujeres también puntúan más alto en hábitos de estudio (HE), así como en las escalas AC, EX, TE y TR. No se han encontrado diferencias de género en el resto de escalas.

\section{Discusión}

En este trabajo se quiso comprobar, tanto la relación entre hábitos de estudio y rendimiento académico, como el análisis de ambos en busca de diferencias por género. Tras realizar una revisión de la literatura al respecto y el análisis estadístico de los datos, los resultados obtenidos 
muestran que, el rendimiento académico está relacionado de forma positiva con los hábitos de estudio en general; esto concuerda con la mayor parte de las investigaciones revisadas (Díaz, 1995; Fullana, 2008; Hernández \& García, 1991; Martínez-Otero, 1996; Meneghetti et al., 2007; Nonis \& Hudson, 2010; Salas, 1999), donde se contempla los hábitos de estudio como una variable determinante del rendimiento académico, incluso como predictora del rendimiento.

Además, al buscar correlaciones entre el rendimiento académico y las distintas escalas que componen el $\mathrm{CHTE}$, encontramos una correlación estadísticamente significativa entre rendimiento académico y las escalas AC (actitud), PL (planificación del tiempo), LU (lugar de estudio), TE (técnicas de estudio) y TR (trabajos). Esto viene a decirnos que dentro de los hábitos de estudio, algo que pueda parecer menos relevante como son, por ejemplo, las condiciones en cuanto al lugar de estudio (LU), está relacionado de forma positiva con el rendimiento académico, o que una buena planificación (PL) también se relaciona con un mejor rendimiento académico, independientemente de las técnicas de estudio que se apliquen o de la actitud que se tenga frente a los estudios.

Son importantes estos datos, pues indican que hay varios aspectos sobre los que incidir de forma más concreta para obtener una mejora en los hábitos de estudio, ya que todos ellos, trabajados por separado o de forma conjunta, son susceptibles de mejora. Por ejemplo, mejorando las condiciones del lugar de estudio podría mejorarse el rendimiento académico de los sujetos. Algunos aspectos referidos al lugar de estudio tienen que ver con la iluminación adecuada, los ruidos que distraen al alumno o a la alumna, la ventilación del lugar de estudio, la altura adecuada de la silla, etc. Muchos sujetos afirmaron estudiar en lugares poco adecuados como la cocina, mientras había ruidos y más personas en la estancia; el sofá, adoptando posturas incómodas que favorecían la fatiga y con la televisión de fondo, aumentando las distracciones. Por ello, es importante mejorar estas condiciones, para que las horas dedicadas al estudio cundan más y consigan así un rendimiento escolar más elevado.

La planificación del tiempo también resulta un aspecto muy importante para estudiantes de estas edades, en esto coincidimos con Corea (2001) y García (1988). En el $2^{\circ}$ Ciclo de ESO las exigencias académicas han aumentado respecto a cursos anteriores y el alumnado se lleva muchas tareas para hacer en casa, además de estudiar para los exámenes. Es necesario pues establecer un plan de trabajo, teniendo las tareas 
Importancia de los hábitos de estudio en el rendimiento académico del adolescente: diferencias por género

Ana Capdevila Seder y Héctor Bellmunt Villalonga

bien organizadas a lo largo de la semana, dándole a cada una de ellas la importancia que se merece. Este plan de trabajo resulta aún más importante si los sujetos realizan actividades extraescolares, como puede ser practicar algún deporte, clases de música, academias, etc., de modo que su tiempo libre se ve reducido, debiendo aprovechar al máximo las pocas horas que puedan dedicar al estudio.

La actitud hacia el estudio es un tema más complejo, que requeriría de un análisis más profundo para averiguar la naturaleza de sus motivaciones o la falta de ellas. Son muchos los alumnos que no saben las razones por las que estudian (este es el primer ítem del cuestionario CHTE), por lo que difícilmente encontrarán la motivación suficiente para hacerlo.

Por otra parte, las escalas ES (estado físico) y EX (exámenes y ejercicios) no se relacionan con el rendimiento académico, cayendo el peso sobre el resto de escalas. Para los autores del CHTE (Álvarez \& Fernández, 2002), la escala que menos contribuye al factor general es la escala ES y en este caso también es la escala que menos influye en el rendimiento académico del alumnado.

Sin embargo, la preparación de exámenes y ejercicios no determina el rendimiento. Resulta Ilamativo este dato, pues la calificación en cada materia viene principalmente de la nota que se obtiene en los exámenes. Quizá el motivo resida en la importancia de las condiciones en que se Ileva a cabo el estudio día tras día: buena actitud, planificación, uso de técnicas de estudio adecuadas o unas óptimas condiciones en cuanto al lugar de estudio, por encima de la preparación de un examen los días previos al mismo.

En cuanto a la puntuación global obtenida en el CHTE, los propios autores del cuestionario proponen un baremo, en el que la puntuación media de la muestra $(66,29$ puntos sobre 100) corresponde a "Estudiante aceptable, podría mejorar en algún aspecto", siendo ésta la tercera mejor valoración posible de entre cuatro propuestas (Álvarez \& Fernández, 2002). Con ello, se puede afirmar que los sujetos estudiados tienen unos hábitos de estudio aceptables pero mejorables.

Por lo que respecta a las diferencias de género, las mujeres, tal y como se ha visto en la introducción, generalmente tienen una nota media superior a los hombres y en este caso se confirma esta hipótesis. Esto concuerda con las investigaciones consultadas (Córdoba, 2010; Corea, 2001; Crosnoe, 2002; Kovacs et al., 2008; Ruiz de Miguel, 2009; Yu et 
al., 2006), donde se concluye diciendo que el género es una variable determinante del rendimiento académico.

En cuanto a los hábitos de estudio, las mujeres obtienen de media hasta 6 puntos (sobre 100) más que los hombres, diferencia significativa estadísticamente hablando. Este dato tiene mucha importancia, pues refuerza la relación positiva obtenida entre las variables RA y HE, como se ha demostrado. El hecho de que las mujeres tengan mejor rendimiento académico y mejores hábitos de estudio no hace más que reafirmar que los hábitos de estudio son un elemento clave en el rendimiento durante la adolescencia.

Además, las mujeres también puntúan más alto que los hombres en AC, EX, TE y TR. Las mujeres tienen mejor actitud que los hombres frente a los estudios, lo que les hace afrontar las tareas académicas con mayor motivación. Según, Amrai et al. (2011), en esto está la clave del éxito académico, pues la motivación académica se relaciona directamente con el rendimiento académico. Para estos autores, a esto hay que sumarle que son precisamente las mujeres quienes mejor preparan los trabajos y los exámenes, parte fundamental en la consecución de buenas calificaciones. Los resultados obtenidos coinciden, además, con esta última afirmación, donde las mujeres han puntuado más alto que los hombres en TR y EX.

Nuestros resultados, en cuanto a género, coinciden con los de Grimes (1985) y con los de Núñez et al. (1995), quienes también obtuvieron mayor puntuación del género femenino en cuanto a actitud y motivación. Sin embargo, a diferencia de los resultados hallados por Grimes (1985), en este caso no se han detectado diferencias en cuanto a la administración de tiempo para el estudio.

$\mathrm{Al}$ analizar las correlaciones entre variables por separado, hombres y mujeres, se observa que en el caso de las mujeres la relación positiva entre RA y HE continúa existiendo, pero entre RA y las escalas del cuestionario CHTE se limita a correlaciones positivas con PL y AC; por lo que a las mujeres parece no influirles en su rendimiento variables como son las condiciones del lugar de estudio, la preparación de trabajos o las técnicas de estudio.

Algo similar ocurre al analizar únicamente a la muestra de hombres, donde además de hallar correlaciones positivas entre RA y HE, PL y AC, también se produce esta relación positiva entre RA y LU, con lo que a los hombres parece sí afectarles las condiciones del lugar de estudio. Habría 
Importancia de los hábitos de estudio en el rendimiento académico del adolescente: diferencias por género

Ana Capdevila Seder y Héctor Bellmunt Villalonga

que profundizar en estos resultados para averiguar el motivo de estas diferencias entre hombres y mujeres, y el por qué a un grupo parece influirles el lugar en el que estudian y al otro no. Aunque una hipótesis podría ser que las mujeres poseen más herramientas frente al estudio y ello hace que aspectos aislados como la comodidad del lugar de estudio o los ruidos, no les afecten tanto, pues compensan estas distracciones con mejor actitud, mejores técnicas de estudio y con una mejor preparación de trabajos y exámenes.

\section{Conclusiones}

Los resultados obtenidos consolidan la afirmación de que el rendimiento académico se relaciona positivamente con los hábitos de estudio, además, es el género femenino quien puntúa más alto en RA y HE.

Los hábitos de estudio son un aspecto fundamental dentro del rendimiento académico. Aprendiendo unos buenos hábitos de estudio el alumnado puede rendir mejor en los estudios. Enseñándoles a planificar el tiempo, a mejorar el lugar y las técnicas de estudio, entre otros aspectos, los sujetos pueden mejorar sus hábitos de estudio y con ello también su rendimiento académico.

Resulta necesario insistir en aplicar programas de mejora de los hábitos de estudio, teniendo en cuenta todos los aspectos que los configuran y no centrándose sólo en las técnicas y métodos de estudio.

Proponemos de cara al futuro aplicar un programa de mejora de los hábitos de estudio, teniendo en cuenta, además de las técnicas de estudio, tanto la preparación de exámenes y trabajos, como la planificación del tiempo, la actitud, el estado físico y las cuestiones relacionadas con el lugar habitual de estudio. Tras este programa se podría comprobar si los sujetos han mejorado sus hábitos de estudio y su rendimiento académico. Habría que insistir más en la población adolescente masculina, puesto que es donde se presenta un peor rendimiento académico y peores hábitos de estudio. 
Importancia de los hábitos de estudio en el rendimiento académico del adolescente: diferencias por género

ana Capdevila Seder y Héctor Bellmunt Villalonga

\section{Referencias bibliográficas}

Álvarez, M. y Fernández, R. (2002). CHTE, Cuestionario de Hábitos y Técnicas de Estudio. Propuesta de un programa de métodos de estudio. Manual. $3^{\text {a }}$ edición revisada y ampliada. Madrid: Tea Ediciones, S.A.

Amrai, K., Motlagh, S.E., Zalani, HA, y Parhon, H. (2011). The relationship between academic motivation and academic achievement students. [doi: 10.1016/j.sbspro.2011.03.111]. Procedia - Social and Behavioral Sciences, 15(0), 399-402.

Baéz, J. y Báez, J.M. (1998). Métodos y técnicas de estudio, manual para estudiantes. Madrid: Edinumen.

Barbero, M.I., Holgado, F.P., Vila, E., y Chacón, S. (2007). Actitudes, hábitos de estudio y rendimiento en Matemáticas: diferencias por género. Psicothema, 19(3), 413-421.

Cartagena, M. (2008). Relación entre la autoeficacia y el rendimiento escolar y los hábitos de estudio en alumnos de Secundaria. Revista Iberoamericana sobre Calidad, Eficacia y Cambio en Educación, 6(3) 60-99. Recuperado de http://www.rinace.net/ arts/vol6num3/art3.pdf

Córdoba, L.G. (2010). Hábitos de estilo de vida en relación con el rendimiento académico en alumnos de la ESO de Extremadura-Badajoz. Universidad de Extremadura, Badajoz.

Corea, N.C. (2001). Régimen de vida de los escolares y rendimiento académico. Universidad Autónoma de Barcelona, Barcelona. Recuperado de http://www.tdx.cat/handle/10803/5002

Crosnoe, R. (2002). Academic and health-related trajectories in adolescence: The intersection of gender and athletics. Journal of Health and Social Behaviour, 43(3), 317335. Tomado el 22/04/2012: http://www.jstor.org/stable/3090207

Díaz, F. (1995). La predicción del rendimiento académico en la Universidad: un ejemplo de aplicación de la regresión múltiple. Enseñanza. Anuario Interuniversitario de Didáctica, 13, 43-61.

Fullana, J. (2008). La investigació sobre l'exit i el fracàs escolar des de la perspectiva dels factors de risc. Implicacions per a la recerca i la pràctica, tesis doctoral, Departament de Pedagogia. Universitat de Girona.

García, V. (1988). Práctica de la educación personalizada. En Cartagena (2008), p.65.

González, C. (2003). Factores determinantes del bajo rendimiento académico en Educación Secundaria. Universidad Complutense de Madrid, Madrid.

Grimes, S.K. (1995). Targeting academic programs to student diversity utilizing learning styles and learning-study strategies. Journal of College Student Development, 36(5), 422-430.

Hernández, P. y García, L.A. (1991). Psicología y enseñanza del estudio. Teorías y técnicas para potenciar las habilidades intelectuales. Madrid: Pirámide.

Kovacs, F.M., Gil, M.T., Gestoso, M., López, J., Mufraggi, N., y Palou, P. (2008). La influencia de los padres sobre el consumo de alcohol y tabaco y otros hábitos de los adolescentes de Palma de Mallorca en 2003. Revista Española de Salud Pública, 82(6), 677-689. Recuperado de http://scielo.isciii.es/scielo.php?pid=S1135$57272008000600008 \&$ script=sci_arttext 
Importancia de los hábitos de estudio en el rendimiento académico del adolescente: diferencias por género

Ana Capdevila Seder y Héctor Bellmunt Villalonga

Martínez-Otero, V. (1996). Factores determinantes del rendimiento académico en enseñanza media. Psicología Educativa, 2(1) 79-90.

Meneghetti, C., De Beni, R., y Cornoldi, C. (2007). Strategic knowledge and consistency in students with good and poor study skills. European Journal of Cognitive Psychology, 19(4/5), 628-649.

Molina, S. y García, E. (1984). El éxito y el fracaso escolar en la E.G.B.Cuadernos de pedagogía. Barcelona: Laia.

Moriana, J.A., Alós, F., Alcalá, R., Pino, M.J., Herruzo, J., y Ruiz, R. (2006). Actividades extraescolares y rendimiento académico en alumnos de Educación Secundaria. Revista Electrónica de Investigación Psicoeducativa, 4(1), 35-46. Recuperado de http:// repositorio.ual.es/jspui/bitstream/10835/622/2/Art_8_82_spa.pdf

Nonis, S.A. y Hudson, G.I. (2010). Performance of Collage Students: Inpact of Study Time and Study Habits. Journal of Education for Business, Mar/Apr, 85(4), 229-238. Recuperado de http://www.tandfonline.com/doi/pdf/10.1080/08832320903449550

Núñez, J.C., González, J.A., García, M.S., González, S., y García, S.I. (1995). Estrategias de aprendizaje en estudiantes de 10 a 14 años y su relación con los procesos de atribución causal, el autoconcepto y las metas de estudio. Revista Galega de Psicopedagoxía, 7(10-11), 219-242.

Perrenoud, P. (1990). La construcción del éxito y del fracaso escolar. Madrid: Morata.

PISA (2012). Programa para la Evaluación Internacional de alumnos de la OCDE. Resumen de los resultados españoles. Nota del País. Recuperado de: https://www. mecd.gob.es/dctm/inee/internacional/pisa2012/nota-pais-esp-ocde-pisa-2012. pdf?documentld $=0901 \mathrm{e} 72 \mathrm{~b} 8178650 \mathrm{~d}$

Plant, E.A., Ericsson, K.A., Hill, L., y Asberg, K. (2005). Why study time does not predict grade point average across college students: Implications of deliberate practice for academic performance. [doi: 10.1016/j.cedpsych.2004.06.001]. Contemporary Educational Psychology, 30(1), 96-116.

Real Academia Española (Ed.) (2007). Diccionario de la Lengua Española (22a ed.).

Ruiz de Miguel, C. (2009). The effective schools: a multinivel study of explanatory factors of the school performance in the area of mathematics. Revista de Educación, 348, 355-376, Enero-Abril.

Salas, M. (1999). Técnicas de estudio para Secundaria y Universidad. Madrid: Alianza Editorial.

SGEEM (2012). Las cifras de la educación en España. Curso 2009-2010. Subdirección General de Estadística y Estudios del Ministerio. Recuperado de http://www.educacion.gob.es/horizontales/estadisticas/indicadores-publicaciones-sintesis/cifras-educacion-espana/2012.html

Wall, W.D. (1970). El Fracaso Escolar. Buenos Aires: Paidos.

Yu, C.C.W., Chan, S., Cheng, F., Sung, R.Y.T., y Hau, K-T. (2006). Are physical activity and academic performance compatible? Academic achievement, conduct, physical activity and self-esteem of Hong Kong Chinese primary school children. Educational Studies, December, 32(4), 331-341. 